\title{
Gestión del talento en turismo
}

\author{
Talent Management in Tourism
}

\author{
Judith Alejandra Velázquez Castro ${ }^{\text {a }}$ Erika Cruz Coria ${ }^{b}$
}

\begin{abstract}
:
Tourism reviews the importance for the development and economic growth of Mexico and other countries. Thus, the World Travel and Tourism Council (WTTC for its acronym in english) notes that the industry employs about 313 million people and gender $10.7 \%$ of the Gross Domestic Product (GDP) worldwide (WTTC, 2017). In the case of Mexico, the sector contributes 8.5\% of GDP to the national economy and more than 4 million jobs (Ministry of Tourism, 2017).

Such diversification is reflected in the globalization of the sector and in the reconfiguration of the tourist offer based on innovation. Therefore, to companies providing tourism services to meet the goals set by the organization; in addition to covering a number of new destinations.

Thus, the tourism industry is faced with the need to contribute to the economic and social development of the country in a sustainable and innovative way, considering the rapid social, cultural and economic transformations in which the involved actors operate; which, the combination generates competitive advantages between destinations, products, companies and companies, both nationally and internationally.

And how to achieve this? Tourism companies have a determining role as responsible for providing tourists with a service that meets their needs and expectations; for this reason, the talent management and the professionalization of personnel is preponderant. Lastly, it should be noted that one of the key aspects for the competitiveness of a destination is the ability of human resources to offer what is required.
\end{abstract}

\section{Keywords:}

Talent management, tourism

\section{Resumen:}

El turismo reviste importancia para el desarrollo y crecimiento económico de México y otros países. De este modo, el Consejo Mundial de Viajes y Turismo (WTTC por sus siglas en inglés) señala que la industria emplea alrededor de 313 millones de personas y genera $10.7 \%$ del Producto Interno Bruto (PIB) mundial (WTTC, 2017). En el caso de México, el sector aporta a la economía nacional 8.5\% del PIB y más de 4 millones de empleos (Secretaria de Turismo, 2017).

Tal diversificación, se ve reflejada en la mundialización del sector y en la reconfiguración de la oferta turística en función de la innovación. Por lo anterior, a las empresas prestadoras de servicios turísticos les corresponde hacer un manejo eficiente del talento, ya que éste es de suma importancia para alcanzar las metas planteadas por la organización; además de que abarca un creciente número de nuevos destinos.

Es así, que la industria turística se enfrenta a la necesidad de contribuir al desarrollo económico y social del país de forma sustentable e innovadora, atendiendo las rápidas transformaciones sociales, culturales y económicas en las que se desenvuelven los actores involucrados; lo cual, permitirá generar ventajas competitivas entre los destinos, los productos, los servicios y las empresas, tanto a nivel nacional como internacional.

¿Y cómo lograr esto? Las empresas turísticas poseen un papel determinante como encargadas de proporcionar a los turistas un servicio que satisfaga sus necesidades y expectativas; por tal razón, la gestión del talento y la profesionalización del personal es preponderante. En último lugar, cabe destacar que uno de los aspectos clave para la competitividad de un destino es, la capacidad de sus recursos humanos para ofrecer lo que se requiere.

\section{Palabras Clave:}

Gestión del talento, turismo

\footnotetext{
a Autor de Correspondencia, Universidad Autónoma del Estado de Hidalgo, Instituto de Ciencias Económico Administrativas, https://orcid.org/ 0000-0001-7138-9293, Email: judithalejandra666@gmail.com

${ }^{\text {b } U n i v e r s i d a d ~ A u t o ́ n o m a ~ d e l ~ E s t a d o ~ d e ~ H i d a l g o, ~ I n s t i t u t o ~ d e ~ C i e n c i a s ~ E c o n o ́ m i c o ~ A d m i n i s t r a t i v a s, ~ E m a i l: ~ e c o r i a 84 @ h o t m a i l . c o m ~}$
} 


\section{GESTION DELTALENTO EN}
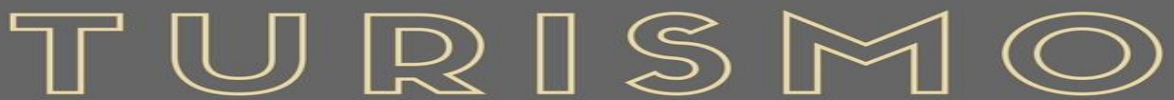

La industria turística emplea alrededor de

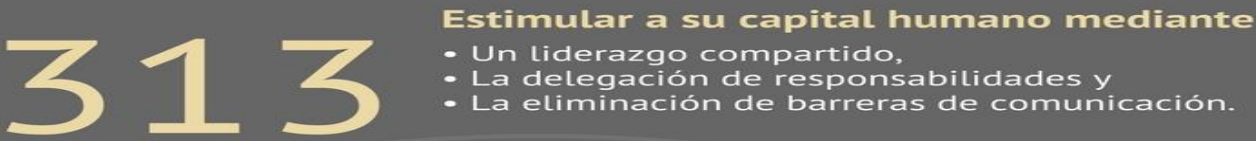

Millones de personas

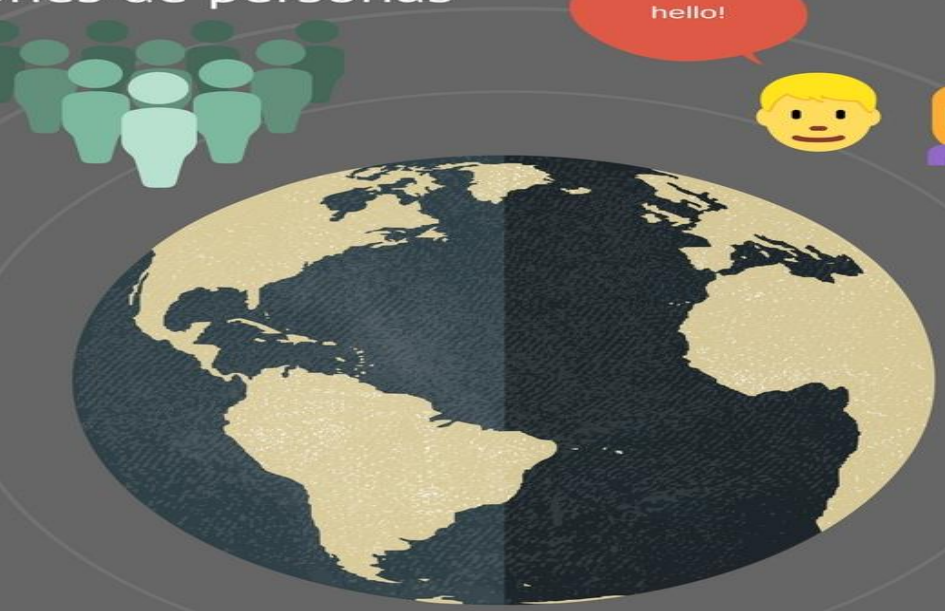

1

La competitividad del sector turístico está condicionada por la importancia que se le otorgue a sus recursos humanos.

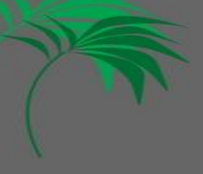

la gestión del talento y la profesionalización del personal es preponderante, ya que son aspectos clave para la competitividad de un destino.

\section{2}

Del sector educativo, dependerá la formación de capital humano de calidad.

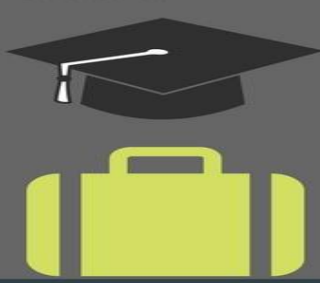

El profesional en turismo deberá comprometerse en su formación constante, ya que al igual que en otras

profesiones, debe mantenerse actualizado e informado de los nuevos acontecimientos que puedan surgir en el sector.

\section{Referencias}

[1] Secretaria de Turismo, SECTUR (2017). Empleo turístico. Consultado el 6 de abril de 2018, de: http://www.datatur.sectur.gob.mx/SitePages/ResultadosITET.aspx

[2] World Travel \& Tourism Council, WTTC (2017). Country economic impact analysis. Consultado el 6 de abril de 2018, de: https://www.wttc.org/research/economic-research/economic-impact-analysis/ 